\title{
Spectroscopy of the nonlinear refractive index of colloidal PbSe nanocrystals
}

\author{
I. Moreels ${ }^{a)}$ and Z. Hens \\ Physics and Chemistry of Nanostructures, Ghent University, B-9000 Ghent, Belgium \\ P. Kockaert \\ Optics and Acoustics Department, Université Libre de Bruxelles, B-1050 Brussels, Belgium \\ J. Loicq \\ Centre Spatial de Liège, Université de Liège, B-4031 Angleur, Belgium \\ D. Van Thourhout \\ Department of Information Technology, Ghent University-IMEC, B-9000 Ghent, Belgium
}

(Received 5 May 2006; accepted 30 September 2006; published online 7 November 2006)

\begin{abstract}
A spectroscopic study of the optical nonlinearity of PbSe colloidal solutions was performed with the $Z$-scan technique at wavelength intervals of 1200-1350 and 1540-1750 nm. While nonlinear absorption remains below the detection threshold, the third order nonlinear refractive index $n_{2}$ shows clear resonances, somewhat blueshifted relative to the exciton transitions in the absorbance spectrum. The occurrence of thermal effects is ruled out by time-resolved measurements. At $1.55 \mu \mathrm{m}$, measured (resonant) $n_{2}$ values exceed typical bulk semiconductor values by two orders of magnitude. At high optical intensity, the refractive index change saturates, indicating that statefilling lies at the origin of the observed effect. (C) 2006 American Institute of Physics.
\end{abstract}

[DOI: $10.1063 / 1.2385658]$

Inorganic nanocrystals make up a new class of materials since, unlike bulk materials, their optical and electronic properties can be effectively engineered by choosing the appropriate material and size of the particles. Over the last 15 years, colloid chemistry techniques have been developed that enable the synthesis of inorganic nanocrystals as highly monodisperse colloidal dispersions, offering great flexibility and easy processing. Applications of colloidal semiconductor nanocrystals (or quantum dots) have already been explored in optoelectronic or photovoltaic devices (photodetectors, ${ }^{1}$ light emitting diodes, ${ }^{2}$ and lasers ${ }^{3}$ ). An additional field of applications may follow from recent studies indicating that quantum dots show strongly enhanced nonlinear optical properties, ${ }^{4}$ and behave as saturable absorbers. ${ }^{5,6}$ Materials with a large and fast nonlinear refractive index can be used in devices such as Mach-Zehnder interferometers, ${ }^{7}$ and absorption saturation is a necessary element in passive modelocked lasers. ${ }^{8}$ Unfortunately, most studies on the nonlinear optical properties of colloidal nanocrystals are performed either at energies well below the first one-photon transition (also referred to as the band gap) ${ }^{4,5}$ or when performed around the band gap, yield results cloaked by thermal effects. ${ }^{9,10}$

In this letter, we present the results of a very detailed study on the nonlinear refractive index $n_{2}$ of colloidal $\mathrm{PbSe}$ quantum dots $(Q$-PbSe $)$ in the near-infrared wavelength region of $1200-1750 \mathrm{~nm}$. Using $Q$-PbSe sizes varying between 3.9 and $5.8 \mathrm{~nm}$, we measured $n_{2}$ as a function of wavelength $\lambda$ and optical intensity $I_{0}$. Covering a spectral range from the transparency region to the second exciton transition, we observed a strong enhancement of $n_{2}$, correlated with the exciton transitions in the absorbance spectrum. Maximal $n_{2}$ values corresponded to -3 to $-410^{-11} \mathrm{~cm}^{2} / \mathrm{W}$

${ }^{a)}$ Electronic mail: iwan.moreels@ugent.be for $1 \mu \mathrm{M}$ suspensions. We observed a saturation of the refractive index $\delta n=n_{2} I_{0}$ only at optical intensities well above the single exciton saturation intensity. This may indicate that the nonlinear refraction is largely due to biexciton creation.

State-of-the-art wet chemical synthesis is currently capable of yielding $Q$ - $\mathrm{PbSe}$ particle dispersions with sizes varying from approximately 2 to $16 \mathrm{~nm}$, all with size distributions $\sigma$ of less than $5 \%{ }^{11,12}$ We optimized the synthesis developed by Murray et al. ${ }^{12}$ to yield monodisperse $Q$ -PbSe solutions $(\sigma<5 \%)$ capped with oleic acid. The characteristics of the samples used for the present study are summarized in Table I. After synthesis, the particles were dispersed in $\mathrm{CCl}_{4}$. The nonlinear refractive index $n_{2}$ and nonlinear absorption $\beta$ were determined with the $Z$-scan technique $^{13}$ using a tunable femtosecond mode-locked laser operating at an $82 \mathrm{MHz}$ repetition rate and at wavelengths in the ranges of $1200-1350$ and $1540-1750 \mathrm{~nm}$. The beam had a Gaussian spatial and a $\operatorname{sech}^{2}$ temporal profile. The beam waist at the focus of the Gaussian beam $w_{0}\left(61 \mu \mathrm{m}^{2}\right)$ and corresponding Rayleigh length $z_{R}(7.8 \mathrm{~mm})$ remained constant throughout the spectral region studied, with deviations occurring only near the edges (at 1200 and $1750 \mathrm{~nm}$ ). Detection thresholds of our setup (determined at

TABLE I. Size and concentration of the three samples prepared. $\lambda_{0}$ denotes the wavelength of the first one-photon transition. Sizes and size distributions were determined using an experimental sizing curve (Ref. 14). Concentrations were determined by weighing the carefully dried samples, taking the weight of the capping molecules into account.

\begin{tabular}{cccc}
\hline \hline Sample & Size $(\mathrm{nm})$ & Concentration $(\mu \mathrm{M})$ & $\lambda_{0}(\mathrm{~nm})$ \\
\hline $\mathrm{A}$ & 5.83 & 0.53 & 1693 \\
$\mathrm{~B}$ & 5.21 & 0.86 & 1555 \\
$\mathrm{C}$ & 3.93 & 1.10 & 1245 \\
\hline \hline
\end{tabular}



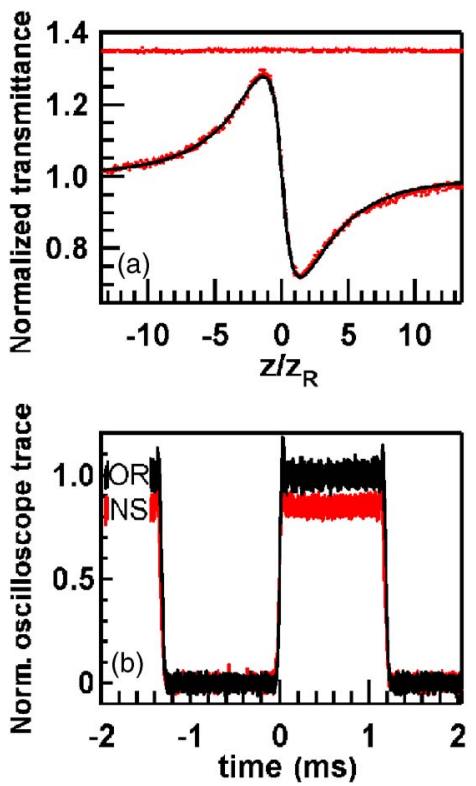

FIG. 1. (Color online) (a) Typical $Z$-scan trace, measured on sample A ( $\lambda$ $=1640 \mathrm{~nm}, I_{0}=11.1 \mathrm{MW} / \mathrm{cm}^{2}$ ). The closed aperture trace (bottom curve) indicates a negative $n_{2}$. The open aperture trace (top curve, offset for clarity) shows no sign of nonlinear absorption. (b) Oscilloscope traces $(\lambda$ $=1693 \mathrm{~nm}$ ) without the sample (NS) and with sample A at the maximum of the closed aperture $Z$-scan trace (OR). Both traces show the same fast buildup of the signal.

$I_{0}=100 \mathrm{MW} / \mathrm{cm}^{2}$ ) corresponded to $n_{2}=10^{-13} \mathrm{~cm}^{2} / \mathrm{W}$ and $\beta=0.5 \mathrm{~cm} / \mathrm{GW}$.

Throughout the entire spectral range, a flat $Z$-scan curve was obtained with $\mathrm{CCl}_{4}$, implying that $n_{2}$ and $\beta$ values remain below the detection threshold. On the other hand, strong nonlinear effects are immediately noticed with $Q$ -PbSe dispersions. Figure 1(a) shows a typical normalized transmittance (closed and open aperture), measured on sample A $\left(\lambda=1640 \mathrm{~nm}, I_{0}=11.1 \mathrm{MW} / \mathrm{cm}^{2}\right)$. The closed aperture trace shows a peak followed by a valley, implying a negative (self-defocussing) $n_{2}$. By fitting the closed aperture trace to a $Z$-scan model trace, ${ }^{14}$ we obtain $n_{2}=-3.010^{-11} \mathrm{~cm}^{2} / \mathrm{W}$. The open aperture trace remains featureless, which means that $\beta$ is less than $4.2 \mathrm{~cm} / \mathrm{GW}$ (the higher threshold results from the lower optical intensity used).

Figure 1(b) represents the oscilloscope trace of the detector intensity obtained by exposing sample A to a chopped laser beam (chopping frequency: $400 \mathrm{~Hz}$; duty cycle: 50\%; wavelength: $1693 \mathrm{~nm}$ ). The sample has been positioned at the maximum of the closed aperture Z-scan trace. Except for a scaling factor due to nonlinear refraction, the trace with the sample (OR) is identical to the trace without the sample (NS). This means that the buildup of the nonlinear signal is faster than the rise time of the chopped beam $(43 \mu \mathrm{s})$. Measurements at $1640 \mathrm{~nm}$ yield the same results. We conclude that thermal lensing, which has a rise time $t_{c}=w_{0}^{2} / 4 D$ of $12 \mathrm{~ms}$ ( $D$ : diffusivity of $\mathrm{CCl}_{4}$ ), can be excluded as the origin of the nonlinear refraction observed.

Figure 2(a)-2(c) displays the results of $Z$-scan measurements performed at wavelengths between 1200 and $1750 \mathrm{~nm}$ for three different samples, covering a spectral range from full transparency to the second exciton transition. Throughout the measurements, $\beta$ always fell below the detection limit, so we will refer only to nonlinear refraction from here on. For all samples, a qualitatively similar $n_{2}$ spectrum is measured. At energies below the first exciton transition, $n_{2}$ remains below the detection threshold. Near the first exciton transition, a clear resonance in $n_{2}$ can be seen. The resonance is slightly blueshifted relative to the maximum of the exciton transition and has a width comparable to that of the (heterogeneously broadened) exciton transition. Rather than the typical dispersionlike $n_{2}$ spectra predicted by theoretical calculations on semiconductor quantum dots, ${ }^{15,16}$ the resonance has a bell shape. For samples A and B, a similar, slightly blueshifted, bell-shaped resonance occurs at the energies of the second exciton transition. For sample $\mathrm{C}$, the second exciton transition cannot be addressed with the setup used. The dependence of the refractive index change $\delta n=n_{2} I_{0}$ on optical intensity is represented in Fig. 2(d) (sample A, $\lambda$ $=1640 \mathrm{~nm}$ ). The figure shows a typical saturation behavior: a linear increase at low intensities levels off towards a constant value at higher optical intensities. A fit to a saturation curve yields a saturation intensity $I_{S}$ of $39.6 \mathrm{MW} / \mathrm{cm}^{2}$.

Depending on the sample, maximal $n_{2}$ values fall in the range of -3 to $-410^{-11} \mathrm{~cm}^{2} / \mathrm{W}$. These values exceed typical
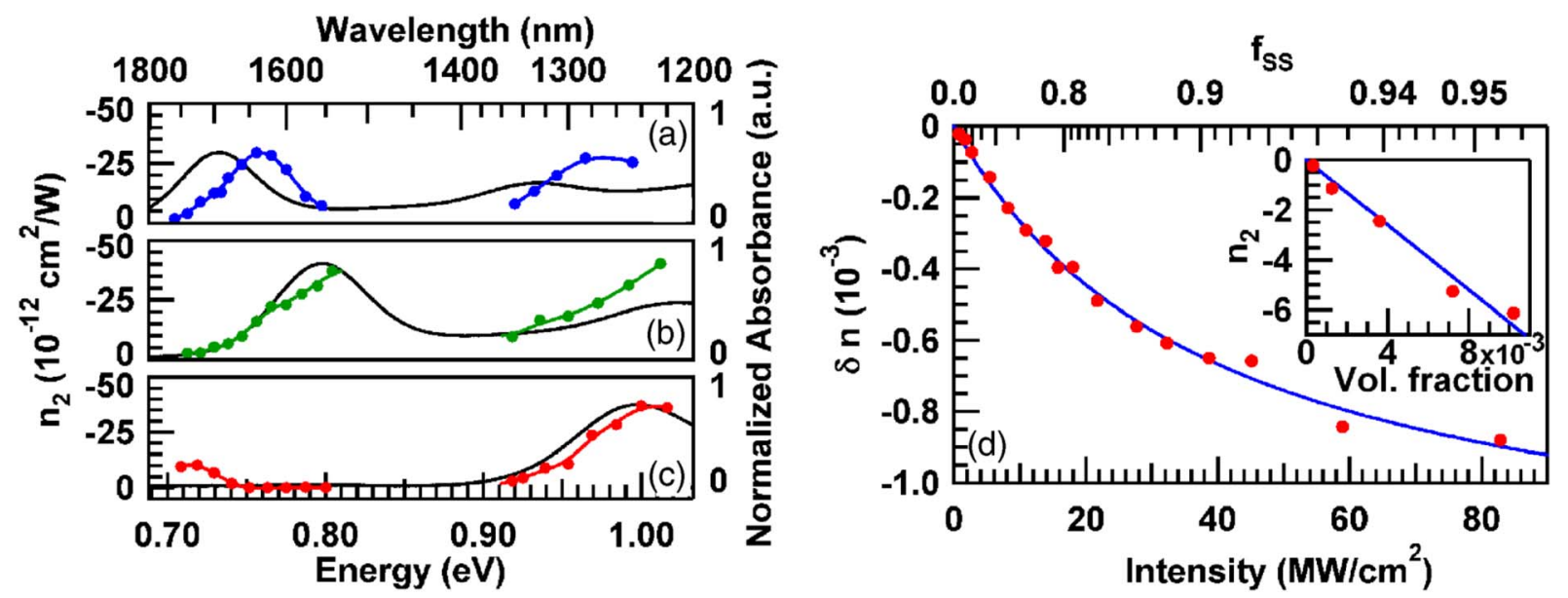

FIG. 2. (Color online) [(a)-(c)] Result of the spectroscopy for samples (a) A, (b) B, and (c) C, with $I_{0}=12 \mathrm{MW} / \mathrm{cm}^{2} . n_{2}$ (dots and interpolated curve) correlates well with the linear absorbance (full black curve). The small peak observed in sample $\mathrm{C}$ around $1700 \mathrm{~nm}$ is due to the oleic acid, as confirmed by measurements on $63.4 \mathrm{mM}$ oleic acid in $\mathrm{CCl}_{4}$ solutions (Ref. 14). (d) Change of the refractive index $\delta n=n_{2} I_{0}$, measured on sample A at $1640 \mathrm{~nm}$. Inset: Measured $n_{2}\left(10^{-11} \mathrm{~cm}^{2} / \mathrm{W}\right)$ vs volume fraction (\%) of the nanocrystals (sample A, $\lambda=1640 \mathrm{~nm}, I_{0}=12 \mathrm{MW} / \mathrm{cm}^{2}$ ).

Downloaded 08 Nov 2006 to 131.211.42.10. Redistribution subject to AlP license or copyright, see http://apl.aip.org/apl/copyright.jsp 
bulk semiconductor values at telecom wavelengths by two orders of magnitude ${ }^{17}$ (measurements on bulk $\mathrm{Si}$ and GaAs at $\lambda=1540 \mathrm{~nm}$ give an $n_{2}$ of $0.4510^{-13}$ and $1.5910^{-13} \mathrm{~cm}^{2} / \mathrm{W}$, respectively). More important for optical signal handling applications is the trade-off between the absorption $\alpha+\beta I_{0}(\alpha$ : linear absorption) and nonlinear refraction, as expressed by the figure of merit $F=n_{2} I_{0} /(\lambda(\alpha$ $\left.\left.+\beta I_{0}\right)\right)$. Setting $\beta$ equal to the detection threshold, a lower limit of $\sim 12.5$ is obtained for suspension B around $1550 \mathrm{~nm}$. This value is significantly better than that of $\mathrm{Si}(0.37), \mathrm{GaAs}$ (0.10), and even $\mathrm{Al}_{0.18} \mathrm{Ga}_{0.84} \mathrm{As}(3.3)$ at $1540 \mathrm{~nm} .{ }^{17,18}$ As the $n_{2}$ values pertain to dilute dispersions of $Q$-PbSe, they scale linearly with the quantum dot concentration as demonstrated in the inset of Fig. 2(d). To compare our results to other materials, we write the measured nonlinear refractive index as the product of the nonlinear refractive index of $Q-\mathrm{PbSe}$ $n_{2, \mathrm{PbSe}}$ and the $Q$-PbSe volume fraction $f$. From the slope of the fitted line in Fig. 2(d), we obtain $n_{2, \mathrm{PbSe}}$ values of $-6.510^{-7} \mathrm{~cm}^{2} / \mathrm{W}$. These are among the highest values reported for colloidal semiconductor quantum dots. $4,5,9,10$

The correlation between the absorbance and the nonlinear refractive index spectrum and the observation of refractive index saturation suggest that state filling lies at the origin of the observed nonlinear effects. Combining the pulse duration, intensity at the focus, and repetition rate of the laser with the lifetime of a single exciton $(0.66 \mu$ s for $5.4 \mathrm{~nm} Q$-PbSe, determined from the luminescence decay), the steady state fraction of nanocrystals containing a single exciton can be calculated. ${ }^{14}$ The respective values have been indicated in the top axis of Fig. 2(d). As the exciton decay rate is far lower than the laser repetition rate $(82 \mathrm{MHz})$, it follows that relatively low intensities lead to high fractions of excited quantum dots. However, more important are the following observations: (1) $\delta n$ keeps increasing markedly even at fractions of excited quantum dots as high as 0.8 or 0.9 and (2) the measured saturation intensity of $39.6 \mathrm{MW} / \mathrm{cm}^{2}$ exceeds the saturation intensity calculated for the first exciton transition $\left(4.0 \mathrm{MW} / \mathrm{cm}^{2}\right)$ by an order of magnitude. This suggests that the main contribution to the nonlinear refractive index observed comes not from the creation of single excitons in unexcited quantum dots. As our spectroscopic measurements have been performed under conditions where up to $75 \%$ or more of the PbSe quantum dots are excited in steady state, creation of biexcitons in already excited quantum dots might cause the observed nonlinear refraction. This suggestion is supported by timeresolved optical bleaching experiments. ${ }^{19}$ These measurements demonstrate that at high optical intensities, the bleaching shows a two component decay: a fast, picosecond component due to the decay of biexcitons and a slow component due to the decay of the single exciton. The short biexciton lifetime (picosecond range) ensures that no biexcitons are present at the start of each laser pulse; this complies with the higher than expected saturation intensity.

In conclusion, we have performed a full spectroscopic study on the nonlinear refractive index of dilute suspensions of $\mathrm{PbSe}$ quantum dots. It is demonstrated that $n_{2}$ shows negative, bell-shaped resonances, slightly blueshifted relative to resonances in the linear absorbance spectrum. At telecom wavelengths, the (resonant) nonlinear refractive index of the
$Q$-PbSe suspensions exceeds that of typical bulk semiconductors by two orders of magnitude. Time-resolved measurements enabled us to exclude thermal nonlinearities. By varying the beam intensity, we demonstrated saturation behavior of the refractive index change of the $Q-\mathrm{PbSe}$. However, the saturation intensity is larger than expected. Considering the results of the intensity-dependent measurements compared to calculations of the saturation intensity, we suggest that the creation of biexcitons might lead to the large nonlinearities observed. By tuning the size of the $Q-\mathrm{PbSe}$, one can easily achieve high nonlinear refractive indices with a high figure of merit at any technologically important wavelength in the NIR. This flexibility, combined with the convenient processing of colloidal nanocrystals, demonstrates that $Q$-PbSe is a promising material in nonlinear applications at telecom wavelengths.

The authors acknowledge the Institute for the Promotion of Innovation through Science and Technology in Flanders (IWT-Vlaanderen) for the scholarship for one of the authors (I.M.), and the Fund for Scientific Research Flanders (FWOVlaanderen) for a research grant to another author (Z.H.) (1.5.089.03). This work was also supported by the Belgian Science Policy Office under Grant No. IAP-V18 to two of the authors (P.K. and D.V.T.). They thank D. Poelman for the use of his spectrophotometer.

${ }^{1}$ D. F. Qi, M. Fischbein, M. Drndic, and S. Selmic, Appl. Phys. Lett. 86, 093103 (2005).

${ }^{2}$ J. Roither, W. Heiss, D. V. Talapin, N. Gaponik, and A. Eychmuller, Appl. Phys. Lett. 84, 2223 (2004).

${ }^{3}$ H. J. Eisler, V. C. Sundar, M. G. Bawendi, M. Walsh, H. I. Smith, and V. Klimov, Appl. Phys. Lett. 80, 4614 (2002)

${ }^{4}$ X. C. Wu, R. Y. Wang, B. S. Zou, P. F. Wu, L. Wang, J. R. Xu, and W. Huang, Appl. Phys. Lett. 71, 2097 (1997).

${ }^{5}$ Y. C. Ker, J. H. Lin, and W. F. Hsieh, Jpn. J. Appl. Phys., Part 1 42, 1258 (2003).

${ }^{6}$ J. He, W. Ji, G. H. Ma, S. H. Tang, H. I. Elim, W. X. Sun, Z. H. Zhang, and W. S. Chin, J. Appl. Phys. 95, 6381 (2004).

${ }^{7}$ R. Prasanth, J. E. M. Haverkort, A. Deepthy, E. W. Bogaart, J. van der Tol, E. A. Patent, G. Zhao, Q. Gong, P. J. van Veldhoven, R. Notzel, and J. H. Wolter, Appl. Phys. Lett. 84, 4059 (2004).

${ }^{8}$ P. T. Guerreiro, S. Ten, N. F. Borrelli, J. Butty, G. E. Jabbour, and N. Peyghambarian, Appl. Phys. Lett. 71, 1595 (1997).

${ }^{9}$ I. Gerdova and A. Hache, Opt. Commun. 246, 205 (2005).

${ }^{10}$ J. Loicq, Y. Renotte, J. L. Delplancke, and Y. Lion, New J. Phys. 6, 32 (2004).

${ }^{11}$ J. M. Pietryga, R. D. Schaller, D. Werder, M. H. Stewart, V. I. Klimov, and J. A. Hollingsworth, J. Am. Chem. Soc. 126, 11752 (2004).

${ }^{12}$ C. B. Murray, S. H. Sun, W. Gaschler, H. Doyle, T. A. Betley, and C. R. Kagan, IBM J. Res. Dev. 45, 47 (2001).

${ }^{13}$ M. Sheikbahae, A. A. Said, T. H. Wei, D. J. Hagan, and E. W. Vanstryland, IEEE J. Quantum Electron. 26, 760 (1990).

${ }^{14}$ See EPAPS Document No. E-APPLAB-89-204645 for sizing curve, fitting procedure, calculation of the saturation intensity, and $n_{2}$ spectrum of oleic acid. This document can be reached via a direct link in the online article's HTML reference section or via the EPAPS homepage (http://www.aip.org/ pubservs/epaps. html).

${ }^{15}$ T. Takagahara, Phys. Rev. B 36, 9293 (1987).

${ }^{16}$ L. Banyai, Y. Z. Hu, M. Lindberg, and S. W. Koch, Phys. Rev. B 38, 8142 (1988).

${ }^{17}$ M. Dinu, F. Quochi, and H. Garcia, Appl. Phys. Lett. 82, 2954 (2003).

${ }^{18}$ A. Villeneuve, C. C. Yang, G. I. Stegeman, C. H. Lin, and H. H. Lin, Appl. Phys. Lett. 62, 2465 (1993).

${ }^{19}$ R. D. Schaller, M. A. Petruska, and V. I. Klimov, J. Phys. Chem. B 107, 13765 (2003). 